\title{
La relevancia de los complejos conceptuales en publicidad
}

The relevance of conceptual complexes for advertising

\author{
Paula PÉrez Sobrino \\ Universidad de La Rioja, España, paula.perezs@unirioja.es
}

\section{Resumen}

Se busca demostrar que las inferencias derivadas de entornos multimodales, como el publicitario, pueden ser dirigidas y constreñidas por un conjunto finito de operaciones cognitivas (en este caso, metáfora en interacción con metonimia). Se exploran varias estrategias de construcción de significado en ejemplos de publicidad impresa, en los que se analizan cuatro cuestiones concretas: (1) ¿cómo contribuye la interacción entre el modo visual y el textual a la identificación de la metáfora y/o la metonimia?; (2) ¿cómo interaccionan la metáfora y la metonimia?; (3) ¿de qué modo esta interacción pauta la interpretación del anuncio?; y (4) ¿de qué forma este complejo conceptual multimodal refuerza y expande el impacto comunicativo del anuncio? Estos procesos de (re)construcción de significado en contextos multimodales son: metonimia, complejo metonímico, metáfora, metaftonimia y amalgama metafórica. Los creativos publicitarios recurren a menudo a estos mecanismos de forma aleatoria e inconsciente. Sin embargo, la incorporación consciente de estas operaciones en las rutinas publicitarias puede ayudar a los anunciantes a lanzar campañas más efectivas y a controlar la forma en que sus audiencias interpretan los mensajes publicitarios; y a los documentalistas a analizar y representar el contenido de los anuncios con más eficacia.
\end{abstract}

Palabras clave: Metáfora. Metonimia. Multimodalidad. Publicidad. Patrones de interacción.

\section{Introducción}

La tesis principal de este trabajo es que la aplicación selectiva de una serie de operaciones cognitivas (metonimia, complejo metonímico, metáfora, metaftonimia, y amalgama metafórica) en el entorno publicitario puede contribuir asegurar la correcta interpretación del mensaje persuasivo por parte de los consumidores, a la par que puede minimizar la generación de interpretaciones no deseadas o negativas. Aunque estos mecanismos son a menudo incorporados en la creación de campañas publicitarias de forma aleatoria e inconsciente, la planificación y uso controlado de estos mecanismos conceptuales por parte de los creativos publicitarios puede ayudar a los anunciantes a crear campañas más efectivas y a controlar la forma en que

\begin{abstract}
This article aims to show that the array of inferences triggered in multimodal environments, such as advertising, can be steered and constrained by a finite set of cognitive operations (in this case, metaphor in interaction with metonymy). In this article I explore several meaning construction strategies in several examples of printed advertising, in which I analyze four specific issues: (1) How does the interplay between the textual and the visual mode contribute to the identification of the metaphor/metonymy? (2) How do metaphor and metonymy interact? (3) How does this interaction cue the interpretation of the advertisement? And (4) how does this multimodal conceptual complex reinforce and expand the communicative impact of the advertisement? These meaning construction processes in multimodal contexts are: metonymy, metonymic complex, metaphor, metaphton$y m y$, and metaphoric amalgam. The conscious use of these mechanisms by the creative team can help advertisers to release more effective campaigns and to control the manner in their audiences interpret their messages; in turn, information scientists can analyse and represent the advertisements more effectively.
\end{abstract}

Keywords: Advertising. Metaphor. Metonymy. Multimodality. Patterns of interaction.

sus audiencias interpretan los mensajes publicitarios; y a los documentalistas publicitarios a analizar y representar más efectivamente el contenido de los anuncios. En este trabajo se exploran las cinco estrategias de construcción de significado multimodal arriba mencionadas en ejemplos de publicidad impresa, con el objetivo de demostrar la forma en la que fijan la atención del consumidor y dirigen la interpretación del anuncio de acuerdo con la intención del anunciante. Cabe destacar que, aun con todo, existen limitaciones de tipo cultural que pueden afectar al funcionamiento de estas operaciones cognitivas y por tanto variar la significatividad de una campaña publicitaria de una sociedad a otra.

En la siguiente sección se revisa brevemente la literatura existente sobre metáfora y metonimia 
multimodal y sus patrones de interacción en publicidad. Fruto del análisis de ejemplos reales de publicidad impresa, se detectan al menos cinco patrones de interacción diferenciados (metonimia, complejo metonímico, metáfora, metaftonimia, y amalgama metafórica). En la sección de análisis se da cuenta de la forma en la que dichos patrones captan la atención de la audiencia, y de cómo dirigen y constriñen la interpretación adecuada del anuncio (a la par que descartan la actividad inferencial no deseada). Por último, se recuperan las propuestas ofrecidas en este trabajo y se apuntan futuras líneas de investigación que surgen de esta investigación.

\section{Metáfora multimodal en publicidad}

En Lingüística Cognitiva, una metáfora es definida como un mapeo conceptual entre dos dominios por la que un dominio (fuente) estructura parcialmente nuestra comprensión de otro (meta) (pueden encontrarse descripciones más detalladas en Gibbs, 1994; Lakoff, 1993; Lakoff and Johnson, 1980, 1999). Lakoff y Johnson (1980) defienden que la metáfora existe a nivel lingüístico precisamente porque refleja cómo nuestro pensamiento está estructurado metafóricamente. Considere, por ejemplo, las múltiples realizaciones lingüísticas de la metáfora conceptual UNA DISCUSIÓN ES UNA GUERRA (Lakoff y Johnson, 1980 , p. 40): "Tus afirmaciones son indefendibles", "Atacó todos los puntos débiles de mi argumento", "Sus críticas dieron justo en el blanco", "Destruí su argumento", “¿No estás de acuerdo? Vale, ¡dispara!" (se ha mantenido la cursiva original para señalar la manifestación lingüística de la metáfora conceptual). Como se infiere del contexto, estas expresiones se enmarcan en el ámbito del debate intelectual, y no el de una batalla real. Sin embargo, nuestro conocimiento enciclopédico de las guerras (dominio metafórico fuente) se recupera parcialmente para estructurar nuestra noción sobre las discusiones (dominio metafórico meta). La metáfora es, pues, un fenómeno fundamental y universal que da forma a nuestras experiencias sociales, intelectuales y emocionales cotidianas.

En los últimos años, la comunidad de lingüistas cognitivos ha sido testigo del creciente interés por la naturaleza, funcionamiento y limitaciones de la metáfora multimodal como mecanismo cognitivo. Este interés está del todo justificado por una de las máximas de la Teoría Conceptual de la Metáfora (Lakoff y Johnson, 1980, p. 153), que establece que "la metáfora es principalmente una cuestión de pensamiento y acción, y de forma derivada de lenguaje". De acuerdo con la definición proporcionada por Forceville (2009a, p. 24), un metáfora multimodal es aquella "metáfora cuyos dominios fuente y meta están representados exclusivamente o predominantemente en diferentes modos". Aunque existe un gran debate en torno a la noción de "modo" (el lector se puede referir a Kress y Leeuwen, 2001, p. 20) (1), en el marco de los análisis de metáfora multimodal en publicidad se refiere principalmente al texto y a las imágenes ( $y$ al sonido o la música en anuncios televisivos).

De esta manera, los estudios en metáfora multimodal pretenden compensar la atención casi exclusiva que se ha dedicado en los últimos treinta años a las manifestaciones lingüísticas de metáforas conceptuales proporcionando ejemplos en otros contextos, como el visual (Forceville, 1996; Forceville y Uriós Aparisi, 2009; y las referencias que contienen Hidalgo y Kralievic 2011), el auditivo (Pérez Sobrino, 2014, Zbikowski 2002), el olfatorio (Velasco Sacristán y Fuertes Olivera, 2006) y el gestual (Cienki y Müller, 2008).

Los expertos en metáfora multimodal encuentran una rica fuente de ejemplos en los entornos publicitarios. De hecho, metáfora y publicidad coexisten de forma natural puesto que la lógica interna de la metáfora como operación cognitiva se ajusta perfectamente a las convenciones de la publicidad como género: ambas se basan en la adscripción de los atributos de un dominio concreto sobre otro (en el caso de la metáfora, del dominio fuente sobre el meta; en el caso de la publicidad, de los valores deseados sobre el producto anunciado).

Desafortunadamente, tanto el análisis de los patrones de interacción conceptual como el análisis de la metonimia multimodal en publicidad no ha atraído la suficiente atención por parte de la comunidad lingüística — si bien con algunas excepciones como Forceville (2009b) y Villacañas y White (2013) para el estudio de metonimias visuales y Pérez Sobrino (2014) para metaftonimia verbomusical en música clásica y contemporánea)—. Se espera que con este artículo se contribuya a crear interés por analizar este fenómeno relativamente inexplorado a día de hoy.

\section{Complejos multimodales en publicidad}

La estructuración de mensajes publicitarios sobre la base de operaciones cognitivas (bien de forma individual o en forma de complejo conceptual) permite a los anunciantes generar mensajes persuasivos que vayan más allá de la estricta representación literal sus anuncios. En efecto, el anuncio se presenta como un punto de acceso económico a todo un conjunto de 
inferencias determinadas por la operación cognitiva subyacente. Dado que la producción inferencial es limitada y está constreñida por la naturaleza de la operación cognitiva que la activa, Pérez Hernández (2011, p. 371) anticipa que la incorporación consciente de estos mecanismos conceptuales en las rutinas publicitarias se presenta como una gran oportunidad para los equipos creativos y de marketing para anticipar el tipo de inferencias positivas que sus anuncios van a generar, a la vez que les ahorra interpretaciones desviadas o erróneas de sus anuncios.

Sin embargo, los profesionales de la industria publicitaria deben ser además conscientes del papel que juega el entorno lingüístico y cultural, que puede bloquear la interpretación del anuncio en los términos pretendidos (o incluso generar mensajes equívocos o negativos) en determinados países.

A continuación se presentan cinco estrategias de construcción de significado multimodal (metonimia, complejo metonímico, metáfora, metaftonimia y amalgama metafórica) en varios ejemplos de publicidad impresa. En concreto, se analizan las siguientes cuatro cuestiones: (1) ¿cómo contribuye la interacción entre el modo visual y el textual a la identificación de la metáfora y/o la metonimia?; (2) ¿cómo interaccionan la metáfora y la metonimia?; (3) ¿de qué modo esta interacción pauta la interpretación del anuncio?; y (4) ¿de qué forma esta operación cognitiva multimodal refuerza y expande el impacto comunicativo del anuncio?

Los anuncios que se analizan pertenecen a una colección de 150 anuncios impresos que contienen algún tipo de uso de lenguaje figurado. Dado que no existe ningún tipo de búsqueda automatizada de este tipo de mensajes multimodales, los anuncios se han recuperado manualmente (y siguiendo la intuición del analista) de www.coloribus.com, www.adsoftheworld.com y Google Images. Los ejemplos que se presentan a continuación se han seleccionado porque reflejan de una forma suficientemente ilustrativa el funcionamiento de los patrones conceptuales que se analizan.

\subsection{Metonimia multimodal}

La metonimia es una operación cognitiva por la que un subdominio (el vehículo) proporciona acceso mental a otro subdominio (el meta) dentro del mismo dominio conceptual (Barcelona, 2000; Kövecses and Radden, 1998; Ruiz de Mendoza, 2013). En una redefinición de la teoría, Ruiz de Mendoza (2000) distingue dos tipos de metonimia: (1) metonimia de vehículo en meta, en la que todo el dominio matriz designa a uno de sus dominios por medio de un proceso de reducción metonímica (como en "se está tomando la píldora", en donde "píldora" designa a la "píldora anticonceptiva"); y (2) metonimia de meta en vehículo, en donde un subdominio designa a todo el dominio conceptual por medio de un proceso de expansión metonímica (como en "manos a la obra", en donde "manos" designa al trabajo llevado a cabo por las manos de los trabajadores, puesto que las manos juegan un papel esencial en el dominio del trabajo). Este último tipo de metonimia es particularmente relevante dentro del terreno publicitario, dado que un anuncio ofrece un punto de acceso económico a un mensaje persuasivo más amplio y complejo que la lectura literaria del anuncio. Considere el siguiente ejemplo (Figura 1).

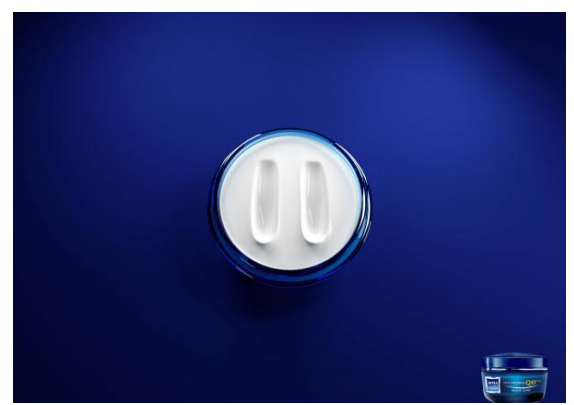

Figura 1. Crema de noche Nivea Q10 antiarrugas

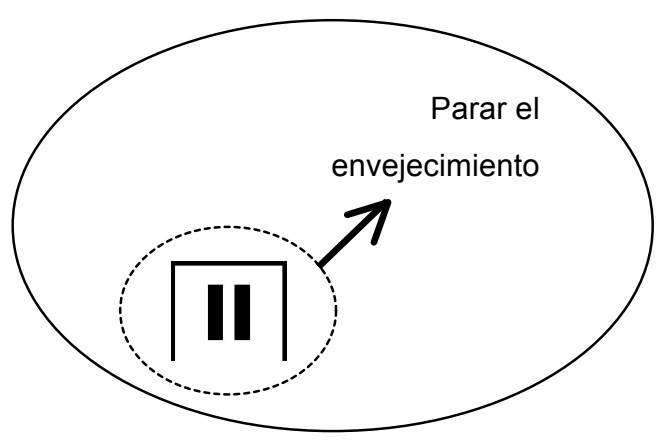

Figura 2. Representación esquemática de la metonimia SímBOLO POR ACCIÓN

La parte visual de este anuncio muestra un tarro abierto de crema sobre un fondo azul oscuro. El tarro muestra las huellas paralelas de dos dedos sobre la crema. Aunque el anuncio no tiene texto, muestra el producto (una crema antiarrugas de noche) en la esquina inferior derecha.

Este ejemplo es particularmente ilustrativo para el estudio del funcionamiento de la metonimia multimodal porque presenta varios mapeos metonímicos simultáneos que desarrollan los diferentes atributos del producto anunciado. La primera metonimia está pautada por las huellas que han dejado los dedos en la superficie de la 
crema, que simulan un símbolo de pausa (tal y como aparece en los reproductores de música y vídeo). Esta marca visual activa la metonimia SíMBOLO POR ACCIÓN, por la que dicho símbolo designa la evento que representa, esto es, la parada de una actividad. Aplicado a este caso en concreto, se trataría de la parada de la acción de envejecer (que es la propiedad principal del ingrediente Q10 que contienen las cremas antiarrugas). Vea una representación esquemática en la Figura 2, en la página anterior.

Paralelamente, la disposición de un círculo blanco (vista superior del tarro de crema abierto) sobre un fondo azul oscuro se asemeja a la imagen de la luna sobre el cielo nocturno. Este escenario activa la metonimia PARTE POR TODO, por el que un elemento constituyente central (la luna) designa un dominio más amplio que lo contiene (la noche), y que además proporciona información adicional con respecto a las instrucciones de uso de la crema (esto es, que es de uso nocturno) (vea la Figura 3).

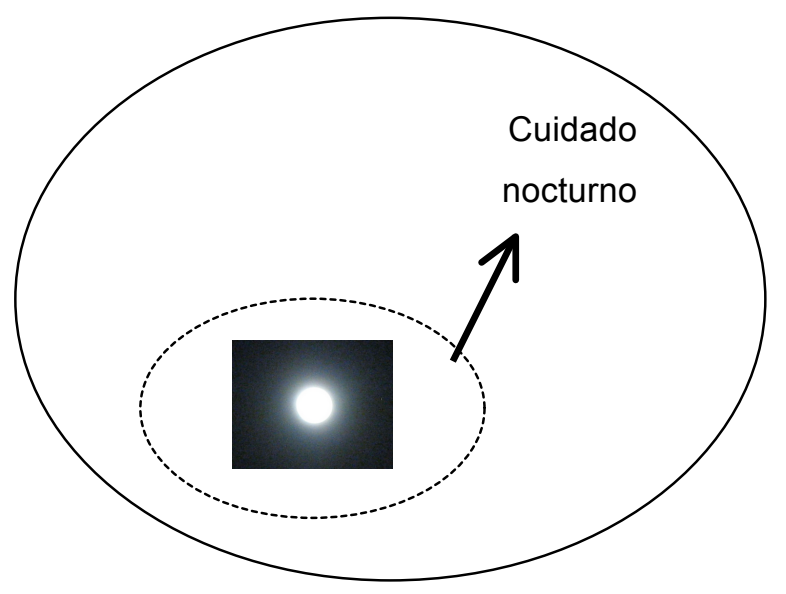

Figura 3. Representación esquemática de la metonimia PARTE POR TODO

Como se puede apreciar por medio de las representaciones gráficas de las metonimias símBOLO POR ACCIÓN Y PARTE POR TODO, ambas reproducen procesos de expansión metonímica. Estas operaciones son especialmente productivas en publicidad (y más aún en anuncios de este corte minimalista), dado que presentan un punto de acceso atractivo y económico (en términos de esfuerzo cognitivo) que facilita el acceso a un mensaje persuasivo más complejo. La parte visual de este anuncio proporciona las claves necesarias para activar una interpretación por parte del consumidor de las características del producto anunciado (en forma de las dos metonimias visuales arriba indicadas) de una forma limitada y predecible.
No obstante, cabe reseñar que los escasos indicios visuales y la ausencia de texto que guíe o refuerce la interpretación también pueden poner en peligro la correcta comprensión del mensaje publicitario para una audiencia poco familiarizada con este tipo de producto. Es posible, pues, que se espere que la audiencia a la que se dirige este anuncio posea un conocimiento previo sobre las propiedades de la vitamina Q10 como agente antienvejecimiento, y que las cremas que contienen este ingrediente son principalmente de aplicación nocturna.

\subsection{Complejo metonímico}

Dos o más metonimias pueden interactuar en cadena, dando lugar a complejos metonímicos (Barcelona, 2005; Brdar-Szabó y Brdar, 2011; Ruiz de Mendoza y Galera, 2014). El efecto comunicativo de este patrón obedece al mismo principio regulador que el de la metonimia simple; pero, obviamente, da lugar a una interpretación más elaborada.

Considere el siguiente anuncio en el que se promociona España como destino turístico (Figura 4).

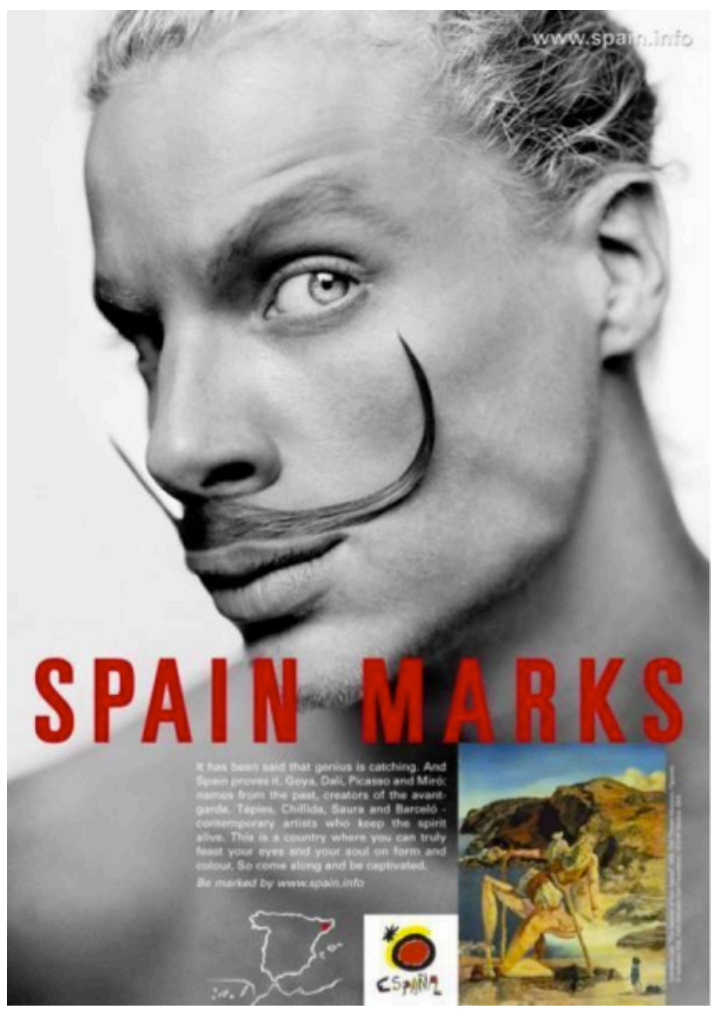

Figura 4. España te marca. Dicen que el genio es contagioso. Y en España hay una verdadera epidemia. Goya, Dalí, Picasso, Miró: nombres de ayer que crearon vanguardias. Tapies, Chillida, Saura,

Barceló: artistas de hoy que mantienen viva la llama. Este es un país donde formas y colores invaden los ojos y el alma. Ven y contágiate. 
Este anuncio presenta a un hombre con un bigote puntiagudo que recuerda al idiosincrásico rasgo del pintor Salvador Dalí. El texto principal reza "España te marca" (en inglés) que junto con el logo de la Marca España revela que se dirige a una audiencia extranjera (presumiblemente, con mayor interés por la cultura que el veraneante de playa) a la que se anima a visitar España.

Para descifrar el sentido de este anuncio, el espectador debe resolver la conexión entre el bigote (el elemento que destaca en la parte visual) y el críptico mensaje textual. Como se ha mencionado, el propio Salvador Dalí hizo de su bigote con puntas hacia arriba su propia marca personal, de tal forma que llegó a ser una característica tan prominente y reconocible de su persona comparable a su propia obra. Así pues, BIGOTE es, en este caso, un elemento definitorio de un dominio conceptual más amplio, SALVADOR DALÍ. Paralelamente, la figura de Dalí es suficientemente representativa como para designar a España como país, en tanto que su pintura es central dentro del patrimonio artístico español e internacional. De esta forma, la interacción de un elemento visual sencillo (bigote) con el texto da lugar a la doble expansión metonímica BIGOTE POR DALÍ POR ESPAÑA, por la que el espectador desarrolla una actividad inferencial que va más allá del anuncio en cuestión: esto es, España es un destino de vacaciones que ofrece un turismo cultural además del de playa, y cuyo patrimonio cultural es tan valioso y definitorio de la identidad de España como lo fue el bigote putiagudo con respecto al propio Dalí. La Figura 5 muestra una representación esquemática de esta doble expansión metonímica.

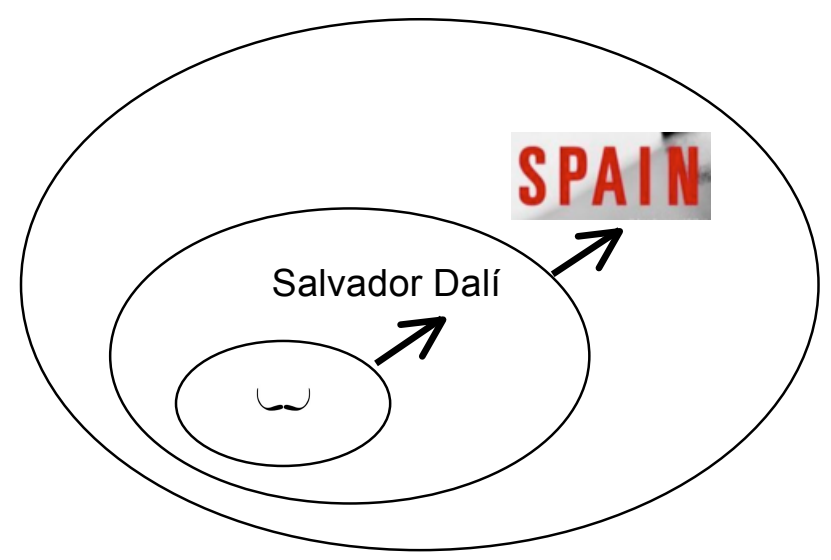

Figura 5. Representación esquemática de la doble expansión metonímica

La afortunada selección del bigote de Dalí como elemento definitorio de Dalí, y por ende, del patrimonio cultural español, facilita la generación de un mensaje positivo que atrae a un tipo de turista diferente al de costa. Además, cabe destacar el papel esencial que juega la parte textual en dirigir la interpretación del producto anunciado como "turismo cultural", a la par que cancela otro tipo de interpretaciones posibles pero desviadas (como la promoción de una exposición de arte surrealista o la promoción de las playas de la Costa Brava).

Podría destacarse como limitación del poder comunicativo del anuncio el hecho de que el espectador debe conocer las características personales del pintor para captar la idea del anuncio. No obstante, puesto que el anuncio pretende promover un turismo más cultural, cabe esperar que la audiencia a la que se dirige cuente con cierto nivel intelectual necesario para llevar a cabo la interpretación del anuncio de forma correcta.

\subsection{Metáfora multimodal}

Como se ha mencionado anteriormente, una metáfora consiste en la puesta en correspondencia de un parte de un dominio (fuente) con otro (meta), en donde ambos dominios están representados total o parcialmente en modos distintos. A modo de ilustración, obsérvese el ejemplo a continuación (Figura 6):

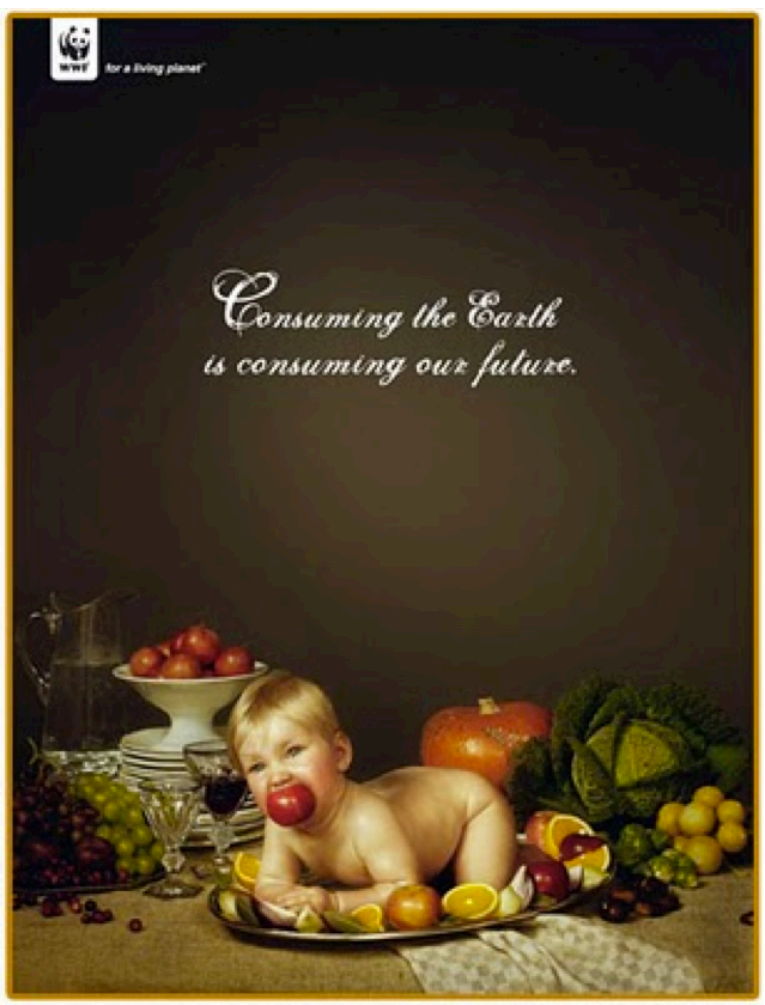

Figura 6. Consumir la Tierra es consumir nuestro futuro 
Un análisis exclusivamente centrado en metáfora visual caracterizaría el bebé (representado en la imagen) y el cochinillo (ausente, pero sugerido por el contexto pictorial) como los dos dominios metafóricos.

Si bien es el dominio fuente el que aparece de forma explícita en las manifestaciones lingüísticas de las metáforas (por ejemplo, en "Lakoff derrotó a Chomsky con sus argumentos", "derrotó" hace referencia al dominio fuente GUERRA, y es el contexto lingüístico el que pauta el dominio meta DISCUSIÓN), esta máxima no se cumple siempre en metáforas multimodales. Para caracterizar ambos dominios como fuente o meta, tanto el analista como el consumidor deben recurrir a la información de contexto que poseen sobre el anunciante. World Wildlife Fund for Nature (WWF) es una organización internacional no gubernamental dedicada a la conservación, investigación y protección del medio ambiente. Así pues, puesto que el objeto de interés de WWF es la protección de las crías animales (y no la promoción del canibalismo), COCHINILLO (ausente) queda establecido como el dominio meta sobre el que se incorporan los atributos característicos del dominio fuente bebé.

De acuerdo con Ruiz de Mendoza y Pérez (2011), las metáforas que ponen en relación a personas con animales (es decir, que están basadas en la Gran Cadena del Ser, Lakoff y Turner, 1989) ponen en correspondencia un atributo central y conspicuo a ambos dominios, que en última instancia es "comportamiento". De tal forma, el comportamiento de un bebé (indefenso y vulnerable) se pone en relación con el de un cochinillo, que por medio de esta metáfora visual se conceptualiza como una víctima de la gula humana, y no como una presa de caza.

\subsection{Metaftonimia}

La literatura existente en metáfora multimodal apenas ha prestado atención a la potencialidad de la metáfora para interactuar con otras operaciones cognitivas (con la excepción de Pérez Sobrino, 2014a; Hidalgo y Kralievic, 2011; y Uriós-Aparisi, 2009). La atención casi exclusiva en el funcionamiento de la metáfora multimodal de forma aislada, si bien justificada por la novedad y el interés generado por el fenómeno, incurre en el riesgo de generar análisis incompletos.

A modo de ilustración, retomemos el caso presentado en la Figura 6. Por el conocimiento previo que el consumidor tiene de WWF, es lógico esperar que, de alguna manera, el mensaje del anuncio se extienda al menos al resto de crías de cerdo, o incluso al conjunto de crías animales. El texto representado en la parte su-

perior del anuncio (que no se tiene en cuenta en el análisis anterior dado que no es una manifestación lingüística de la metáfora, sino una relación de causa-efecto) estipula que es el consumo abusivo de los bienes de la Tierra (y no solo el de crías de animales) el que llevará a la extinción de los humanos (y no solo de los bebés). Es decir, el consumidor debe llevar a cabo un ajuste cognitivo adicional al mapeo metafórico multimodal para entender el significado completo de este anuncio.

En resumen, varias expansiones metonímicas del tipo PARTE POR TODO tanto en el dominio fuente como en el meta (representados visualmente) permiten conectar el cochinillo (ausente en el entorno visual) con el conjunto de crías animales, y posteriormente, con el conjunto de los bienes comestibles de "la Tierra" (textual). De la misma forma, varias expansiones metonímicas hacen del bebé (visual) un punto de acceso económico para acceder un dominio meta más amplio (textual, "el futuro"). La doble expansión metonímica en ambos dominios metafóricos es un ejemplo de metaftonimia (Goossens, 1990; Ruiz de Mendoza y Díez, 2002), esto es, la inclusión de metonimias en sendos dominios metafóricos (vea una representación esquemática de este complejo conceptual en la Figura 7).

FUENTE

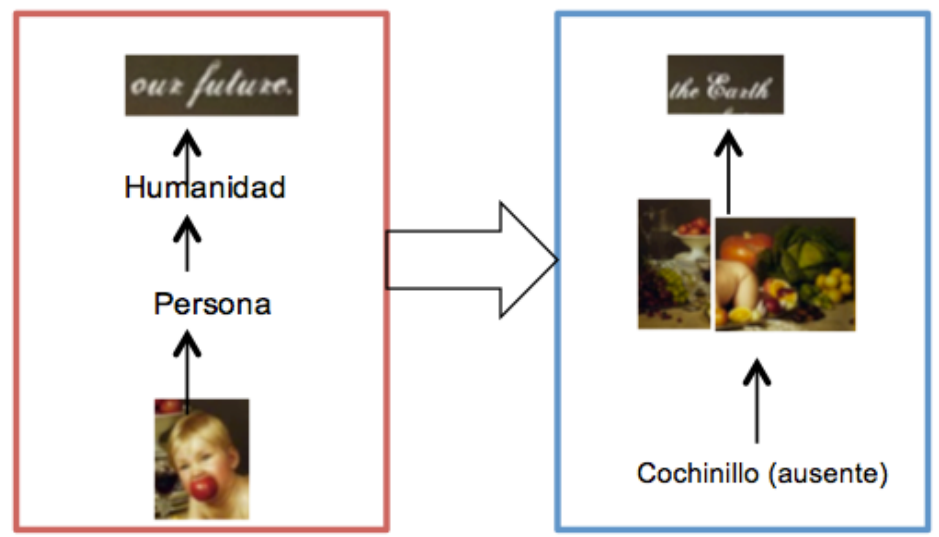

Figura 7. Representación esquemática de los patrones de interacción entre metáfora y metonimia (flechas en negro para los mapeos metonímicos; flechas en blanco para el mapeo metafórico).

Aun con todo, queda por determinar si la combinación de la metáfora con las metonimias realmente garantiza la interpretación correcta del mensaje publicitario. Puesto que el anuncio representa un escenario altamente situacional, la labor de la metonimia aquí es doble. Por un lado, proporciona al consumidor un punto de fácil acceso (un bebé) a un escenario específico 
e incongruente que va a ser empleado para estructurar una situación paralela a la representada (en la que se infiere que es un cochinillo, y no un bebé, el que debería estar sobre plato en medio del bodegón). Por otro, desarrolla la metáfora multimodal en la extensión requerida (determinada por la parte textual) para que el mensaje tenga sentido y el consumidor pueda resolver la incongruencia representada en el anuncio (esto es, la vulnerabilidad de un bebé se mapea no solo sobre el cochinillo, sino sobre todas las crías de animales y posiblemente los recursos naturales de la tierra que necesitamos para vivir).

Tanto los elementos visuales como los textuales son relevantes para la correcta interpretación de este mensaje de una forma natural y previsible. El texto juega un papel crucial a la hora de anticipar las inferencias que van a realizar los consumidores, ya que delimita la elaboración de los dominios fuente y meta metafóricos por medio de expansiones metonímicas.

\subsection{Amalgama metafórica}

Asimismo, dos metáforas pueden interactuar entre sí en forma de amalgama metafórica (Ruiz de Mendoza y Pérez, 2011). Tal y como se ha mostrado para el caso de la metaftonimia, la amalgama metafórica requiere la integración de aspectos específicos de las metáforas involucradas en la interacción. A modo de ilustración, consideremos el análisis del siguiente anuncio de IBM (Figura 8).

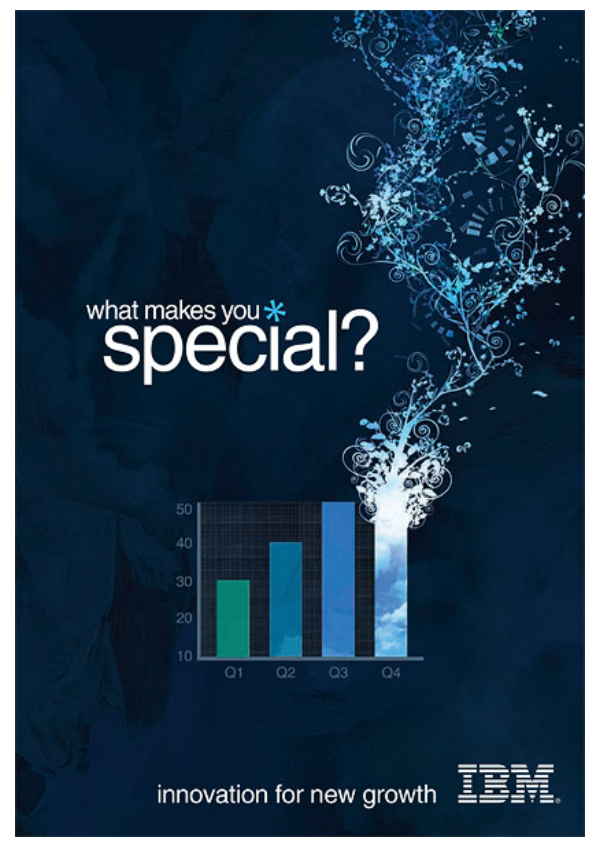

Figura 8. ¿Qué te hace especial? Innovación para un nuevo crecimiento, IBM

A pesar de la aparente simplicidad de este anuncio, el espectador debe disipar una serie de incógnitas para llegar a comprender el mensaje de IBM: ¿qué simbolizan las barras ascendentes? ¿por qué la última barra se sale del marco del anuncio? ¿por qué la última barra tiene formas curvilíneas a partir de cierto punto?¿cuál es la conexión entre el gráfico y la parte textual del anuncio? Como se ha venido demostrando y aquí se pone aún más en evidencia, el uso económico de los elementos visuales y textuales no está necesariamente ligado al volumen y la complejidad de la configuración conceptual que estructura el mensaje.

DOMINIO FUENTE: MOVIMIENTO

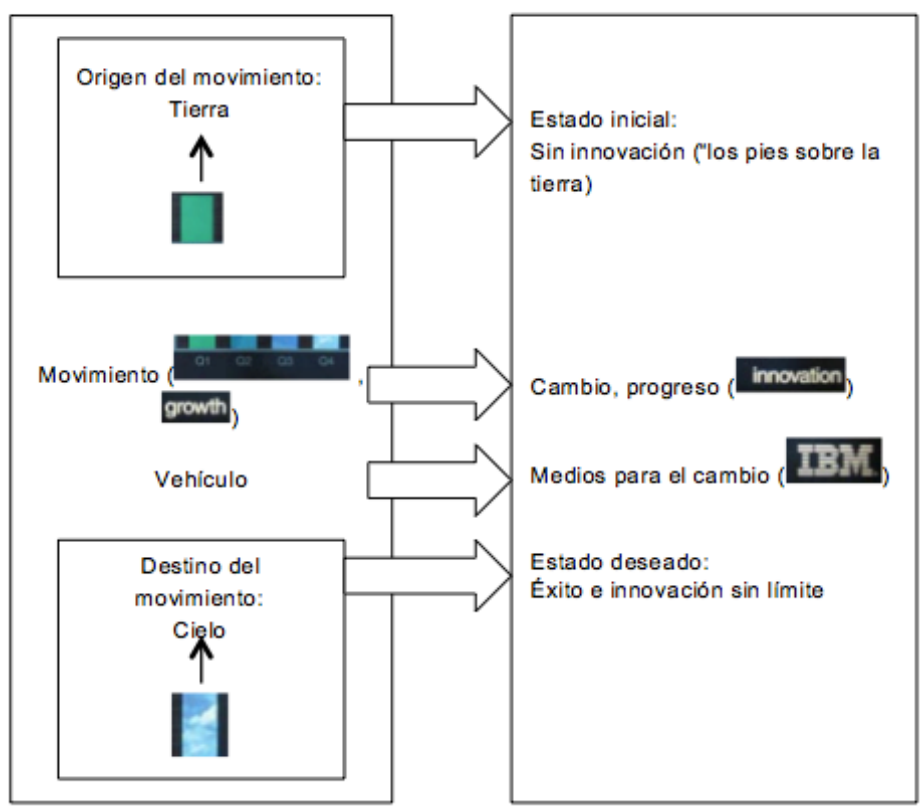

Figura 9. Representación esquemática de la metaftonimia CAMBIO ES MOVIMIENTO (flechas en negro para los mapeos metonímicos; flechas en blanco para el mapeo metafórico)

En este caso, tanto la parte textual "crecimiento" como el gráfico de barras que van creciendo por cuartiles transmiten la idea de movimiento entendido como un cambio de estado entre el primer cuartil (30, según el eje vertical) y el cuarto cuartil (fuera del gráfico y del marco del anuncio). Por medio de la metáfora CAMBIO (DE ESTADO) ES MOVIMIENTO (A LO LARGO DE UN CAMINO), los anunciantes retratan a IBM bien como una empresa en crecimiento (en ventas, en volumen de trabajadores, en visibilidad en mercado, o cualquier otra variable que se pueda representar gráficamente) o como el medio para que otras empresas logren la expansión. Tal y como puede verse en la Figura 9, las correspondencias metafóricas que percibe el consu- 
midor conectarían (a) el estado inicial (la barra del primer cuartil, en donde el color verde es supuestamente metonímico de la tierra o suelo) con un estado primario en donde no hay innovación ni creatividad (como en la expresión "tener los pies sobre la tierra"); (b) la progresión de un cuartil a otro (determinado por cuatro barras ascendentes y la palabra "crecimiento") como un cambio de estado entre un escenario sin innovación y otro con innovación; (c) el vehículo en movimiento a lo largo del camino con el motor del cambio, que en este caso es IBM; y (d) el destino del movimiento con el estado deseado (apuntado visualmente por la barra del último cuartil, que no solo sobrepasa los límites del anuncio, sino que está coloreada de azul cielo con nubes, reforzando la idea de que no hay límite superior que constriña el éxito del cambio alcanzado por IBM).

Curiosamente, una consideración aún más detallada de la parte no verbal del anuncio invita a refinar este análisis preliminar. La información proporcionada por el gráfico es relevante en tanto que incluye una valoración positiva acerca del tipo de cambio y el destino final al que llega IBM por medio de la innovación. La evolución ascendente de las barras, así como la cuarta barra coloreada de color azul cielo con nubes y prolongada más allá del límite superior del marco, activan un segundo mapeo metafórico, Lo BUENO ES ARRIBA. Desde la perspectiva de Lakoff y Johnson (1980), las metáforas basadas en el eje vertical, que son un tipo de metáfora orientacional, proporcionan la base experiencial para conceptualizar el bienestar humano. De acuerdo con esta idea, el movimiento ascendente o una posición superior se corresponden con la postura natural cuando estamos sanos, despiertos y/o felices. Así pues, los estados superiores traen consigo un connotación inherentemente positiva y dan pie a la elaboración de metáforas evaluativas aún más específicas (como por ejemplo, TENER CONTROL O FUERZA ES ARRIBA, CONSCIENTE ES ARRIBA, SALUD Y VIDA ES ARRIBA, que complementan a sus opuestos ESTAR SUJETO A UNA FUERZA O CONTROL ES ABAJO, INSCONSCIENTE ES ABAJO, ENFERMEDAD Y MUERTE ES ABAJO). De esta manera tan sutil, el consumidor que ve un movimiento ascendente percibe no solo un cambio, sino un cambio positivo que conduce al éxito.

La incorporación de la metáfora LO BUENO ES ARRIBA (que es puramente visual) en la estructura preexistente de EL CAMBIO ES MOVIMIENTO (a la que se accede bien por el texto o el gráfico) da lugar a una metáfora más compleja, EL CAMBIO EXITOSO ES MOVIMIENTO ASCENDENTE, por la que en el contexto tecnológico, IBM queda con-

ceptualizada como una empresa que lidera el progreso en la innovación (así referido en el texto) y la creatividad (indicado por la ausencia de límites en la última barra, el color azul cielo, y el diseño creativo de la mitad superior de la barra). Vea una representación esquemática de esta amalgama metafórica en la Figura 10.

DOMINIO FUENTE

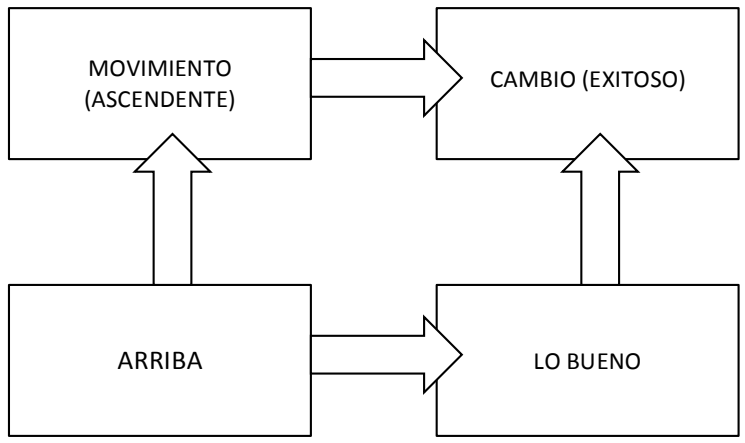

Figura 10. Amalgama metafórica

El cambio exitoso es movimiento ascendente

De forma complementaria, el entramado curvilíneo que conforma la mitad superior de la cuarta barra se asemeja a una estructura orgánica que crece como una planta. Por medio de la metáfora EL DESARROLLO DE UNA IDEA ES EL CRECIMIENTO DE UNA PLANTA (que se manifiesta en ejemplos como "una cultura floreciente" o "una idea enraizada"; Lakoff, 1991, p. 107) se refuerza la idea del crecimiento sin límites de la compañía por medio de la innovación. De esta forma, los anunciantes se aseguran que, bien por medio de la amalgama o por medio de esta segunda metáfora, el consumidor interprete el anuncio en los términos del éxito de IBM como empresa líder en innovación. Más aún, el diseño orgánico de la cuarta barra puede dinamizar la imagen de IBM como una empresa flexible con sus empresarios o incluso preocupada por la conservación del medio ambiente.

\section{Conclusiones}

Un examen detallado de los patrones de interacción conceptual entre la metáfora y la metonimia en publicidad presenta beneficios en una doble vertiente: por un lado, es una oportunidad para los lingüistas cognitivos y documentalistas para explorar maneras detalladas y finitas de explorar la creación de significado multimodal; por otro, sirve de guía para los anunciantes en el diseño de campañas más creativas e impactantes a la par que efectivas y relevantes para sus audiencias. 
Este estudio evidencia la versatilidad de los patrones de interacción conceptual para dar cuenta de mensajes persuasivos multimodales con diferentes grados de complejidad conceptual. La interacción entre la metáfora y los procesos de expansión metonímica permite conseguir un equilibrio óptimo entre la economía en la representación del mensaje, la interpretación que desencadena y el esfuerzo cognitivo requerido para la interpretación. Como si el anuncio se tratara de la punta de un iceberg, los anunciantes solo tienen que representar parte de un escenario más o menos complejo que evoque al consumidor un amplio número de inferencias que le ayuden a recomponer el mensaje persuasivo.

La incorporación de métodos cuantitativos al análisis de corpus multimodales puede abrir nuevas vías de investigación. Futuros estudios deberían indagar en el número de elementos multimodales que pautan el mismo dominio metafórico/metonímico (facilitando así su identificación), el modo (visual o textual) en el que se suelen representar esos dominios, o la frecuencia con la que los dominios fuente y meta están presentes o ausentes en los anuncios. Las respuestas a estas preguntas pueden ser cruciales para desvelar los factores que facilitan o dificultan la correcta interpretación del anuncio por parte de la audiencia otros profesionales.

\section{Nota}

(1) Dado que este trabajo se centra en la exploración de las operaciones cognitivas que motivan la creación y comprensión de una serie de anuncios, y la forma en la que estas operaciones guían la actividad inferencial, los postulados de la Semiótica Social Visual (Kress and Leuween 1996, 2001; Moya y Pinar 2008) quedan desafortunadamente fuera de consideración para el marco teórico, debido a su énfasis en la descripción de la interacción de modos y funciones que estructuran el anuncio.

\section{Agradecimientos}

Este trabajo se ha financiado gracias a una ayuda FPU del Ministerio de Educación de España.

\section{Referencias}

Barcelona, Antonio (2005). The multilevel operation of metonymy in grammar and discourse, with particular attention to metonymic chains. // Ruiz de Mendoza, F. J.; Peña, M. S. (eds.). Cognitive Linguistics: Internal Dynamics and Interdisciplinary Interaction. Berlin: Mouton de Gruyter, 2005. 313-352.

Brdar-Szabó, Rita; Brdar, Mario (2011). What do metonymic chains reveal about the nature of metonymy? // Benczes, R.; Barcelona, A.; Ruiz de Mendoza, F. J. (eds.), Defining Metonymy in Cognitive Linguistics: Towards a consensus view. Amsterdam/Philadelphia: John Benjamins, 2011. 217-248.

Cienki, A; Müller, C. (eds.) (2008). Metaphor and Gesture. Amsterdam: John Benjamins, 2008.
Forceville, Charles (1996). Pictorial Metaphor in Advertising London/New York: Routledge, 1996.

Forceville, Charles (2009a). The role of non-verbal metaphor sound and music in multimodal metaphor. // Forceville, C; Uriós-Aparisi, E. (eds.) Multimodal Metaphor. Berlin/New York: Mouton de Gruyter, 2009. 383-400.

Forceville, Charles (2009b). Metonymy in visual and audiovisual discourse. // Ventola, E.; Moya, A. J. (eds.). The World Told and the World Shown: Multisemiotic Issues. London: Palgrave-McMillan, 2009. 56-74.

Forceville, Ch.; Uriós-Aparisi, E. (eds.) (2009). Multimodal Metaphor. Berlin/New York: Mouton de Gruyter, 2009.

Gibbs, Raymond (1994). The poetics of mind: Figurative thought, language, and understanding. New York: Cambridge University Press, 1994.

Goossens, Louis (1990). Metaphtonymy: The interaction of metaphor and metonymy in expressions for linguistic action. // Cognitive Linguistics 1 (1990). 323-340.

Hidalgo, Laura; Kraljevic, Blanca (2011). Multimodal metonymy and metaphor as complex discourse resources for creativity in ICT advertising discourse. // Gonzálvez García, F.; Peña Cervel, S.; Pérez Hernández, L. (eds.) Metaphor and Metonymy Revisited beyond the Contemporary Theory of Metaphor. Special Issue of the Review of Cognitive Linguistics. 4 (2011). 153-178.

Kövecses, Zoltan; Radden, Günter (1998). Metonymy: Developing a cognitive linguistic view. // Cognitive Linguistics. 9 (1998). 37-77.

Kress, Gunther; van Leeuwen, Theo (2001). Multimodal discourse: The modes and media of contemporary communication. Londres: Arnold, 2001.

Lakoff, George (1991). The Master Metaphor List. http://araw.mede.uic.edu/ alansz/metaphor/METAPHOR LIST.pdf (1 de abril de 2014)

Lakoff, George (1993). The Contemporary Theory of Metaphor. // Ortony, A. (ed.) Metaphor and Thought. Cambridge: Cambridge University Press, 1993. 202-251.

Lakoff, George; Johnson, Mark (1980). Metaphors We Live By. Chicago: University of Chicago Press, 1980.

Lakoff, George; Jonhson, Mark (1999). Philosophy in the Flesh: The Embodied Mind and its Challenge to Western Thought. New York: Basic Books, 1999.

Lakoff George; Turner, Mark (1989). More Than Cool Reason: A Field Guide to Poetic Metaphor. Chicago: University of Chicago Press, 1989.

Pérez Hernández, Lorena (2011). Cognitive Tools for Successful Branding. // Applied Linguistics. 32:4, 369-388.

Pérez-Sobrino, Paula (2014a). Multimodal cognitive operations in classical music. // Vigo International Journal of Applied Linguistics. 11, 137-168.

Pérez-Sobrino, Paula (2014b). Conceptual disintegration and multimodal metonymy in musical understanding. // Journal of Pragmatics. 70, 130-151.

Ruiz de Mendoza, Francisco (2000). The role of mappings and domain in understanding metonymy. // Barcelona, A. (ed.). Metaphor and Metonymy at the Crossroads. Berlin/New York: Mouton de Gruyter, 2000. 109-132.

Ruiz de Mendoza, Francisco y Olga Díez (2002). Patterns of conceptual interaction. // Dirven, R., Pörings, R. (eds.). Metaphor and Metonymy in Comparison and Contrast. Berlin/New York: Mouton de Gruyter, 2002. 489-53. 
Ruiz de Mendoza, Francisco; Galera, Alicia (2014). Cognitive Modeling. A linguistic perspective. Amsterdam/Philadelphia: John Benjamins, 2014.

Ruiz de Mendoza, Francisco; Pérez, Lorena (2011). Conceptual Metaphor Theory: myths, developments and challenges. // Metaphor and Symbol. 26 (2011) 1-25.

Velasco Sacristán, Marisol; Fuertes Olivera, Pedro (2006). Olfactory and olfactory-mixed metaphors in print ads of perfume. // Annual Review of Cognitive Linguistics. 4:1 (2006) 217-252.

Villacañas, Beatriz; White, Michael (2013). Pictorial metonymy as creativity source in "Purificación García" advertising campaigns. // Hidalgo Downing, L.; Kraljevic Mujic, B. (eds.). Metaphorical creativity across modes: Special issue of Metaphor and the Social World. 3:2 (2013). 2013. 220-239.

Zbikowski, Lawrence M (2002). Conceptualizing Music: Cognitive Structure, Theory, and Analysis. New York: Oxford University Press, 2002. AMS Studies in Music.

\section{Referencias de los sitios analizados}

Consuming the Earth is consuming our future. http://adsoftheworld.com/media/print/wwf_baby (consultado el 11 de abril de 2012). Agencia publicitaria: Germaine. Amberes (Bélgica) Fecha: 2008.

Nivea Visage Anti-wrinkle. Q10 Plus Cream. http://www.ad vertolog.com/nivea/print-outdoor/pause-11176655/ (consultado el 1 de marzo de 2014). Agencia publicitaria: Tbwalraad (Emiratos Árabes Unidos). Fecha: 2008.

Spain Marks. http://lapublicidadestaenelaire.blogspot.co.uk/ 2008/10/spain-marks.html (consultado el 1 de marzo de 2014). Agencia publicitaria: Instituto de Turismo de España (Turespaña). Fecha: 2003.

IBM. Website: http://andy-potts.com/work/ibm/ (consultado el 1 de marzo de 2014). Agencia publicitaria: Ogilvy Nueva York (Estados Unidos). Fecha: 2006.

Enviado: 2014-04-17. Segunda versión: 2014-05-31 Aceptado: 2014-07-10. 